\title{
Erratum: Interfacial contributions to spin-orbit torque and magnetoresistance in ferromagnet/heavy-metal bilayers [Phys. Rev. B 101, 020407(R) (2020)]
}

\author{
K. D. Belashchenko (1), Alexey A. Kovalev, and M. van Schilfgaarde \\ (Received 16 November 2021; published 3 December 2021)
}

DOI: 10.1103/PhysRevB.104.219901

In the original paper, the torquances were erroneously reported as functions of the orientation of the spin angular momentum rather than the magnetization $\mathbf{m}$, which is opposite in sign due to the negative gyromagnetic ratio. Consequently, torque components that are odd in $\mathbf{m}$ were reported with the wrong sign. Specifically, the signs are wrong for the fieldlike $C_{1,-1}^{(2)}$ coefficient shown in Fig. 1(b) and the planar-Hall-like $C_{2,1}^{(1)}$ coefficient shown in Fig. 1(a).

This correction does not affect the qualitative conclusions but is important for comparison with experimental data. In particular, the corrected sign of $C_{2,1}^{(1)}$ for $\mathrm{Co} / \mathrm{Pt}$ bilayers agrees with the measurements of Ref. [1] (Ref. [44] in the original paper).

In addition, a factor $l_{s f}$ is missing in the right-hand side of Eq. (4). This factor was correctly included in Fig. 2.

[1] C. Safranski, E. A. Montoya, and I. N. Krivorotov, Nat. Nanotechnol. 14, 27 (2019). 